Review began 08/11/2021 Review ended 08/17/202 Published 08/25/2021

๑ Copyright 2021

is an open access article distributed under the tern of the Cretive distributed under he lerms of the Creative Chen perits unesticted use, distribution, and reproduction in any medium, provided the original author and source are credited.

\title{
The Impact of Peer Support on Patient Outcomes in Adults With Physical Health Conditions: A
}

\section{Scoping Review}

Ellie Grant ${ }^{1}$, Louise Johnson ${ }^{1}$, Apostolos Prodromidis ${ }^{2}$, Peter V. Giannoudis ${ }^{2}$

1. Clinical and Health Psychology, Leeds Teaching Hospitals NHS Trust, Leeds, GBR 2. Academic Department of Trauma and Orthopaedics, University of Leeds, Leeds, GBR

Corresponding author: Ellie Grant, ellie.grant1@nhs.net

\section{Abstract}

Little is known about the impact of peer support programmes on physical health populations or on the methods used to evaluate such programmes. The present study undertakes a scoping review of research related to peer support programmes or interventions in physical health populations, guided by the Preferred Reporting Items for Systematic Reviews and Meta-Analyses extension for scoping reviews (PRISMA-ScR).

The search was carried out across the Medline, PsycINFO, and Cochrane databases and focused on papers that evaluated peer support intervention(s) in adults with physical health conditions. The search identified an initial 7,903 records, which were narrowed down to 21 records that met the inclusion criteria; their findings were narratively synthesized.

The scoping review found considerable heterogeneity among eligible records in terms of their study design, outcome measurements and findings reported. Qualitative methods of evaluation generated more consistent findings compared to objective outcome measures and suggested that peer support was beneficial for patients' health and wellbeing by reducing feelings of isolation and creating a sense of community as well as providing an opportunity for information consolidation. The scoping review highlights the inconsistencies in methods used to evaluate peer support interventions and programmes in healthcare settings among different physical health populations. It also draws attention to the lack of peer support research in particular areas, including in acute physical health populations such as in major trauma. The scoping review emphasizes the need for future studies to address this gap in peer support research.

Categories: Physical Medicine \& Rehabilitation, Psychology, Public Health

Keywords: peer support, patient outcome research, patient-centered outcomes research, rehabilitation psychology, rehabilitation program

\section{Introduction And Background}

'Peer support' is a process that involves individuals drawing on lived experience or shared characteristics to provide knowledge, experience, emotional assistance, practical help, and social interaction to help each other [1]. Peer support can take many forms such as one-to-one, group work, and online and telephone support sessions. It can be informal or more formalised, with trained peer supporters. Peer support in healthcare settings can be standardized by content or function. A global initiative in 2010 that aimed to promote best practices in peer support for health around the world adopted a functional approach to the standardization of peer support and identified the following four core functions: (1) to provide practical support; (2) to provide social and emotional support; (3) to ensure linkages to clinical care and community resources and (4) to provide ongoing support [2].

There is an abundance of peer support research in healthcare that focuses on mental health and HIV, and now more attention is being directed towards alternative population groups, including those with long-term physical health conditions. Research has also shown that peer support is especially valued by young people and British Black, Asian and Minority Ethnic (BAME) adults [3]. Those from "hardly reached" populations have also been found to benefit from peer support interventions, suggesting that peer support is a broad and robust strategy for reaching these groups that health services often fail to engage [4].

There is an increasing evidence base suggesting a range of benefits of peer support for people across various populations. A review commissioned in 2015 that included more than 1,000 research studies concluded that peer support has the potential to improve experience, psycho-social outcomes, behaviour, health outcomes and service use among people with long-term physical and mental health conditions [5]. A further review of the literature showed that peer support leads to significant improvements for people with long-term physical and mental health conditions across various outcomes such as quality of life, social functioning and perceived support, individual's knowledge, skills and confidence to manage their health and care and physical functioning and ability to self-care [6].

The objectives of this scoping review were twofold. First, to gain a better understanding of how various pee support programmes impact patient outcomes in patients with physical health conditions. Second, to gain an insight into how peer support programmes are evaluated. This method of review was selected to enable outcomes to be synthesized in order to provide more context to the evidence base and inform clinical practice. The following research question was generated: What is known about peer support programmes in physical health populations, and how are these programmes evaluated in terms of their effectiveness?

Review

Protocol and registration

The final protocol was registered prospectively with Figshare

(https://figshare.com/articles/preprint/Scoping_review_protocol_The_impact_of_peer_support_on_patient_outcomes_in_adults_with_physical_hea and the review was conducted with reference to this protocol.

\section{Eligibility criteria}

Papers were eligible if they: (1) evaluated a peer support programme in a physical health setting(s) including in primary, secondary or community care settings; (2) included individuals with any physical health condition; (3) included any type of peer support programme/intervention; (5) were published in peerreviewed journals. 


\section{Cureus}

\section{Information sources}

The search was carried out across multiple healthcare databases: Medline (Interface: EBSCOhost), PsycINFO (Interface: Healthcare Databases Advanced Search) and the Central (Interface: Cochrane Library). Search results were imported into a citation manager software (Endnote), and duplicates were removed via a combination of the removal of duplicates function on the programme and a manual check by one of the authors.

\section{Search}

The following search terms were used to search for eligible studies in all databases up to July 012021 . Search terms were intentionally few to reduce the likelihood of omitting papers that may not have specifically indicated including participants from within the broad umbrella of those with physical health conditions but rather mentioned the condition itself. Search terms were limited to within titles and abstracts of studies.

1. Peer support*

AND

2. Evaluation* OR Review*

\section{Selection of sources of evidence}

Screening of papers was guided by the Preferred Reporting Items for Systematic Reviews and Meta-Analyses (PRISMA) framework. One reviewer screened all of the publications, including the title, abstract and full-text screening and was supported by the other reviewers. All reviewers were involved in determining the quality of the screening process and any queries or disagreements were resolved through in-depth discussion.

\section{Data charting process}

Data from selected studies were extracted using a standardized data collection form amended for this review. This tool captured information related to the characteristics of studies, including study aim(s), design, population demographics, nature of intervention(s), description of outcome(s) and method(s) of evaluation of data. One reviewer extracted data from the studies with guidance from the other two reviewers. Data were then added to the characteristics of sources of evidence table (Appendix).

\section{Data items}

Data abstracted included country of origin, population group, type of peer support programme or intervention, including method of delivery, evaluation methods used, main outcomes, including objective measures for quantitative studies, and derived themes for qualitative studies and conclusions.

\section{Synthesis of results}

Studies were grouped by their method of evaluating the peer support programme (either quantitatively or qualitatively). Synthesis of quantitative findings within studies involved summarizing the population group, peer support intervention(s) (including mode of delivery and evaluation methods) and primary outcome measures described. Similar qualitative findings between studies were grouped and over-arching themes are discussed.

\section{Results}

Selection of Sources of Evidence

Following the removal of duplicates, a total of 4,151 studies remained. Figure 1 shows the PRISMA flow diagram used for the identification of eligible studies [7]. 


\section{Cureus}

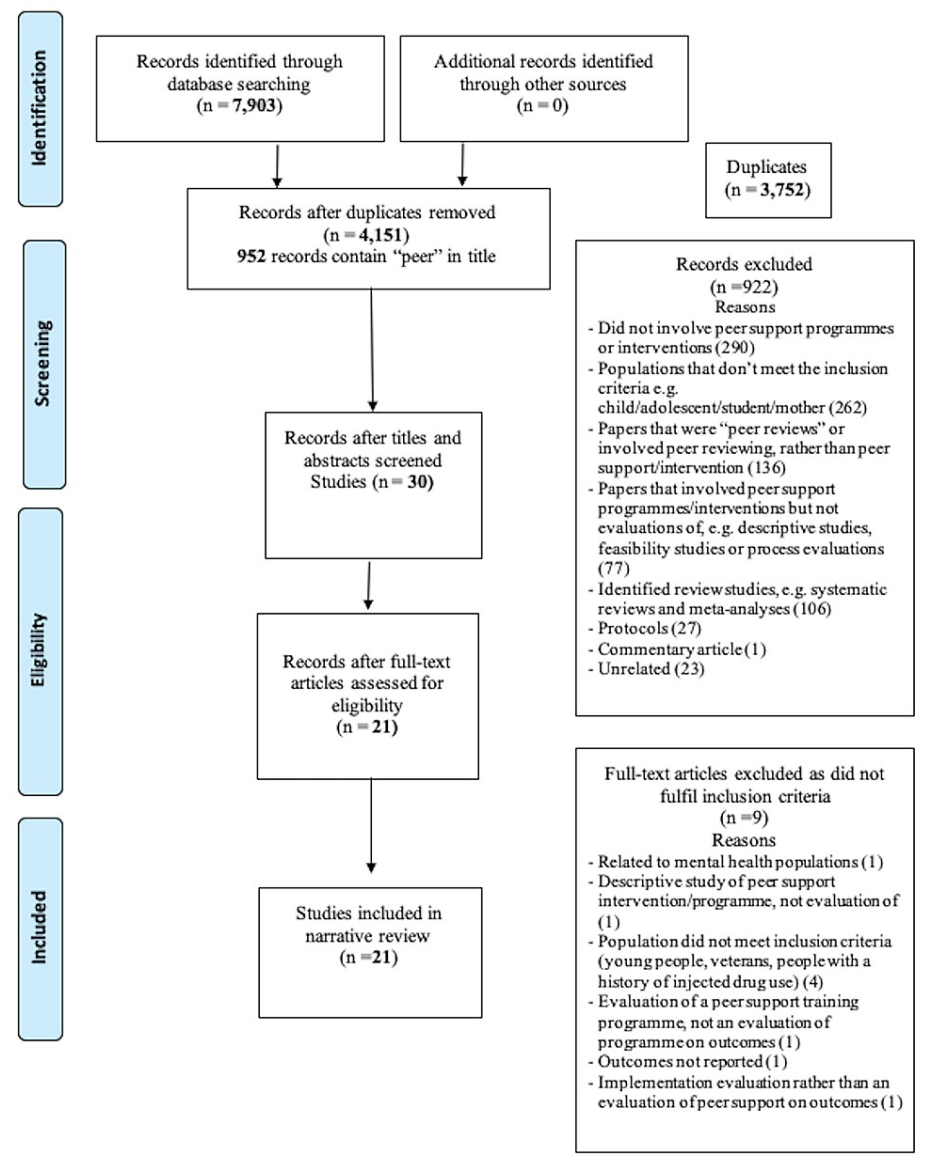

FIGURE 1: PRISMA diagram of included studies

PRISMA: Preferred Reporting Items for Systematic Reviews and Meta-Analyses

Characteristics of Sources of Evidence

Sample sizes of the included studies varied considerably, ranging from eight participants [8] to 1,229 participants [9]. There was considerable heterogeneity in the population groups of included studies. Seven studies included patients with type 2 diabetes (33.3\%) [9-15], five studies included cancer populations (24\%) [8,16-19], two studies included patients with spinal cord injuries (9.5\%) [20-21], seven studies (33.3\%) included participants from other physical health populations; stroke [22], fibromyalgia [23], alopecia [24], polycystic ovary syndrome [25], HIV [26], cardiac [27] and burn injury patients [28].

\section{Peer support interventions}

Mode of Delivery

Table 1 shows variation in studies for the mode of delivery of peer support. 


\section{Cureus}

\section{Author(s) [ID]}

Smith, Paul, Kelly et al., 2011 [11]; Shen, Wang and Edwards, 2017[13]; Ono, Tsuyumu, Ota et al., 2017 [17]; Legg, Occhipinti, Ferguson et al., 2011 [18]

Power and Hegarty, 2010 [8]; Piatt, Rodgers, Xue et al., 2018[15], Clark, Munday and McLaughlin et al., 2012 [27]

Percy, Gibbs, Potter et al., 2009 [25]

Chan, Sui, Oldenburg et al., 2014 [10]; Dale, Caramlau, Sturt et al., 2009[14], St-Pierre, Bouchard, Gauthier et al., 2018 [16]; Gotay, Moinpour, Unger et al., 2007 [19]

Muller, Toth-Cohen and Mulcahey, 2014 [22]

Wingate, Graffy, Holman et al., 2017 [9]; Ayala, Ibarra, Cherrington et al., 2015[12]

Haas, Price and Freeman, 2013[20]; O'Dell, Earle, Rixon et al., 2019[21]; Sallinen, Kukkurainen and Peltokallio et al., 2011 [23]; Iliffe and Thompson, 2019 [24]; Monroe, Nakigozi, Ddaaki et al., 2017 [26]; Grieve, Shapiro, Wibbenmeyer et al., 2020 [28]
$\%$ of Mode of delivery of peer

studies support

Facilitated programme of face-toface peer support led by trained peer supporters

Facilitated programme of face-to-

$14 \%$ face peer support led by bo peer supporters and health professionals

Facilitated programme of peer support led by health professionals alone

Telephone-based peer support programme with trained peer supporters

Broad programme of support with peer support components

Mixed delivery of peer support (telephone and face-to-face)

No peer support intervention but evaluations of previously completed peer support programmes

TABLE 1: Mode of delivery of peer support.

Evaluation Methods

Nine studies (43\%) utilized quantitative means of outcome evaluation in order to determine the efficacy of peer support programmes or interventions [9-11,15,18-19,27-29]. Nine studies (43\%) involved exploratory qualitative analyses of participant perceptions, feedback or experiences of peer support programmes [8,16$17,20-21,23-26]$. Three studies (14\%) incorporated both quantitative and qualitative analyses to evaluate peer support $[13-14,22]$.

Primary Outcomes

Self-report measures used to assess primary outcomes are presented in Table 2. 


\section{Cureus}

\begin{tabular}{|c|c|c|}
\hline Author(s), [ID] & Outcome assessed & Scale/measure used \\
\hline \multicolumn{3}{|l|}{ Psychological } \\
\hline Smith, Paul, Kelly et al., 2011 [11] & Wellbeing & Study does not report on the measure used ${ }^{*}$ \\
\hline $\begin{array}{l}\text { Legg, Occhipinti, Ferguson et al., } \\
2011 \text { [18] }\end{array}$ & Psychological affect & The Hospital and Anxiety Depression Scale (HADS) \\
\hline Shen, Wang and Edwards, $2017[13]$ & Self-efficacy & The Chinese Diabetes Self-Efficacy Scale (C-DSES) \\
\hline $\begin{array}{l}\text { Dale, Caramlau, Sturt et al., } 2009 \\
\text { [14] }\end{array}$ & Self-efficacy & $\begin{array}{l}\text { The Diabetes Management Self-Efficacy Scale } \\
\text { (DMSES) }\end{array}$ \\
\hline Shen, Wang and Edwards, 2017 [13] & Quality of life & $\begin{array}{l}\text { The Medical Outcome Study 36-item Short-Form Health } \\
\text { Survey (SF-36) }\end{array}$ \\
\hline Rodgers, Xue et al., 2011 [15] & Diabetes distress & Diabetes Distress Scale (DDS) \\
\hline $\begin{array}{l}\text { Gotay, Moinpour, Unger et al., } 2007 \\
\text { [19] }\end{array}$ & Psychological distress & $\begin{array}{l}\text { Cancer Rehabilitation Evaluation System-Short-Form } \\
\text { (CARES-SF) }\end{array}$ \\
\hline $\begin{array}{l}\text { Gotay, Moinpour, Unger et al., } 2007 \\
\text { [19] }\end{array}$ & Depressive symptoms & $\begin{array}{l}\text { Center for Epidemiologic Studies Depression Scale } \\
\text { (CES-D) }\end{array}$ \\
\hline \multicolumn{3}{|l|}{ Functional/behavioural/social } \\
\hline $\begin{array}{l}\text { Muller, Toth-Cohen and Mulcahey., } \\
2014 \text { [22] }\end{array}$ & Healthy adjustment after stroke & The Stroke Impact Scale (SIS) \\
\hline $\begin{array}{l}\text { Muller, Toth-Cohen and Mulcahey., } \\
2014 \text { [22] }\end{array}$ & $\begin{array}{l}\text { Home integration, social interaction and } \\
\text { productivity }\end{array}$ & The Community Integration Questionnaire (CIQ) \\
\hline $\begin{array}{l}\text { Grieve, Shapiro, Wibbenmeyer et al., } \\
2020 \text { [28] }\end{array}$ & Social participation & The Life Impact Burn Recovery Evaluation Profile \\
\hline $\begin{array}{l}\text { Clark, Munday and McLaughlin et } \\
\text { al., } 2012 \text { [27] }\end{array}$ & Physical activity level & $\begin{array}{l}\text { 7-day Physical Activity Recall Questionnaire (and } \\
\text { pedometers) }\end{array}$ \\
\hline $\begin{array}{l}\text { Clark, Munday and McLaughlin et } \\
\text { al., } 2012[27]\end{array}$ & Support for physical activity & The Social Support in Exercise Survey \\
\hline Shen, Wang and Edwards, 2017 [13] & Social support & $\begin{array}{l}\text { The Medical Outcome Study Social Support Survey } \\
\text { (MOS-SSS) }\end{array}$ \\
\hline Shen, Wang and Edwards, 2017 [13] & Self-management behaviours & The Chinese Diabetes Self-care Scale (C-DSCS) \\
\hline Rodgers, Xue et al., 2011 [15] & Self-management behaviours & Self-monitoring of blood glucose \\
\hline
\end{tabular}

TABLE 2: Measures used to assess primary outcomes.

*No description of measure used to assess wellbeing in the paper

Psychological Outcomes

The psychological outcome measures cited across included studies varied greatly, with self-efficacy the most commonly reported psychological outcome. A community-based peer-led diabetic self-management programme that involved face-to-face peer support, concluded that self-efficacy significantly improved during the 12-week study period [13]. Notably, the intervention itself centred around self-efficacy enhancing group activities, therefore the programme was tailored to impact this particular outcome. Quality of life among participants, however, did not change significantly. A further study that evaluated a telephone peerdelivered intervention for individuals with type 2 diabetes, found no statistically significant difference in self-efficacy scores at six months [14]. However, multiple differences existed between the studies, despite similarities in the population group and the targeted outcome construct. These differences included the tools used to assess self-efficacy (see Table 2), the delivery of peer support itself (group versus telephone) and the length of time at follow-up (4 versus 6 months).

One study reported a beneficial impact of peer support on diabetes-related distress [15]. In this study, participants were individuals with type 2 diabetes and were randomly allocated to either the intervention group, which involved diabetes self-management education alongside peer-led diabetes self-management support or the Enhanced Usual Care (EUC) group, which was similar to the intervention group, however, it had no components of peer support. The study revealed that peer-led diabetes self-management support was more effective than EUC at improving diabetes distress [15].

One study reported no impact of peer support on psychological outcomes. This study considered the impact of a peer-delivered telephone intervention for women experiencing a breast cancer recurrence [19]. The study concluded that at the three-month follow-up, there were no differences in psychological distress or depressive symptoms between the intervention and control groups.

One study detected a possible detrimental impact of peer support on psychological outcomes for individuals with type 2 diabetes. This randomised controlled trial (RCT) involved a peer-led face-to-face peer support programme that took place over a two-year period. At the two-year follow-up, it was concluded that for the intervention group, there was a reduction in wellbeing (non-significant) compared to the control group [11] Importantly, the measure used to assess wellbeing was not reported in this paper, thereby it is difficult to assess its validity and reliability.

Two studies $[10,18]$ reported that peer support was more effective in psychological outcomes in population groups that experienced higher levels of negative psychological affects compared to those with low levels. The first study involved telephone-based peer-support and reported that participants with greater negative 
emotions seemed to benefit significantly more in terms of their psychological health compared with patients with lower levels of negative emotions. These participants also showed greater treatment compliance and reduced hospital admissions [10]. The second study included a peer-delivered face-to-face peer support programme for individuals with breast cancer and concluded that participants that engaged in positive upward comparison to the breast cancer survivor and who also regarded their cancer diagnosis as more threatening in the first instance, appeared to benefit psychologically by showing lower levels of depressive symptoms [18]. These findings suggest that peer support interventions could be more effective in individuals that demonstrate high levels of negative psychological affect.

Functional, Behavioural and Social Outcomes

The impact of peer support on reported functional, behavioural and social outcomes is mixed. A study that evaluated long-term social reintegration outcomes for burn survivors concluded that those who reported peer support attendance had better social interaction scores than those who did not. This included fewer restrictions in participating in social activities, relating and maintaining friendships and dealing with strangers [28]. A study that evaluated a community-based peer-led diabetic self-management programme concluded that social support and self-management behaviours improved significantly during the 12 -week study period [13]. Another study revealed that peer-led diabetes self-management support was as effective as Enhanced Usual Care in helping participants to maintain self-monitoring of their blood glucose levels [15].

In comparison, a longitudinal study that involved a facilitator-led programme of face-to-face peer support alongside group-based education for people with heart disease who had completed centre-based cardiac rehabilitation reported that social support did not change between baseline and 12-month follow-up [27]. Additionally, there were no changes in the amount of physical activity reported among programme participants.

\section{Physical Health Outcomes}

One study in this review reported a positive impact of peer support on physical health outcomes in individuals with uncontrolled diabetes [12]. A mixed peer-delivered peer support programme concluded that peer support was effective at reducing glycated haemoglobin in intervention versus usual care arm participants [12].

In contrast, an RCT that evaluated the effect of a telephone-based peer support programme in patients with type 2 diabetes found that peer support did not improve cardiometabolic wellbeing [10]. Another study involving participants with type 2 diabetes that utilized a programme of peer-led face-to-face peer support concluded that peer support did not significantly improve physical outcomes (haemoglobin levels, systolic blood pressure and cholesterol levels) [11].

Economic Outcomes

An economic evaluation of a mixed delivery peer support intervention concluded that peer support was associated with lower overall total healthcare costs, which were largely due to a decrease in hospitalization expenses [9]. The evaluation also found that the intervention was associated with a modest increase in outof-pocket costs for participants and implementation costs. The authors suggested that the increase in outof-pocket costs may be explained by an increase in participants' engagement in self-care activities. This study was the only one included in the review that considered economic outcomes following peer support therefore there is a limited evidence base to validate its findings. Moreover, this study reported findings over a relatively short time period (8-12 months).

Qualitative Findings

Some participants described peer support as "a significant turning point in their lives" [23] and something that had a "major personal impact" [25]. Some participants reported that peer support allowed them to "dare to be oneself" [23] whilst others expressed their view that peer support is essential to patients who undergo rehabilitation in a general hospital [20]. A common theme reported among the included studies was peer support leading to a sense of belonging or feeling as though participants were part of a community, which led to reduced isolation and feeling understood $[13,16-17,23]$. Another key theme was peer support as a means of providing and/or consolidating information $[8,22,26]$. Peer support proved helpful for some participants in their decision-making $[8,16]$; others described peer support as responsible for feelings of empowerment $[23,25]$ as well as helpful in aiding self-management behaviours [25].

Much of the qualitative findings suggest that peer support can be beneficial for individuals across various population groups. However, some limitations of peer support were discussed among these positive findings. For example, one study reported that participants experienced raised anxiety related to the future, occasional hopelessness and despair as a result of seeing others with more severe functional disabilities [23]. Furthermore, participants with spinal cord injuries and healthcare practitioners reported in a study that the timing of peer support is essential, as it is not always feasible to deliver in the acute stages of injury [21]. Some practical limitations of peer support programmes were offered, notably matching limitations, strict management of personal information [17] and participants wanting more time to engage in peer support [8].

\section{Discussion}

The heterogeneity in findings within the included studies is clear across the various outcomes, namely, psychological, functional/social/behavioural, health and economic. Studies that included qualitative analysis as their method of evaluating peer support programmes appear to offer more consistency in terms of findings, with most suggesting a beneficial impact for participants. This could mean that the impact of peer support is experienced by participants in various settings, however, the actual impact may not be observable or measurable.

There was considerable variation in the methods used for the delivery of peer support in the included studies. It is therefore difficult to review study findings as a collective. Some interventions focused predominantly on the delivery of peer support as its main component, whilst other interventions focused heavily on education methods with additional peer support. Consequently, it would not be appropriate to attribute peer support as the main influencer of outcomes.

Noteworthy, the majority of included studies did not report any long-term outcomes of associated peer 


\section{Cureus}

support interventions. One study that did, discussed negative feelings reported by participants as a result of seeing others with more severe functional disabilities [23]. This suggests that the functional capabilities of peer supporters as well as the level of exposure to other peers with more severe disabilities need to be considered when developing a peer support programme. Future studies aiming to evaluate peer support programmes should seek to include longitudinal follow-up outcome measures.

Studies that included a measurable peer support component ranged considerably in terms of the length of time of the delivery of peer support, from four weeks [19] to two point eight (2.8) years [16]. Attendance in peer support programmes/interventions was episodic and the number of peer support sessions within the study duration differed enormously. For example, one study [22] reported a sample size of 13 , however, only three (23\%) participants attended all of the described peer support sessions.

There was considerable variation in the measures used to quantify the impact of peer support. For example, the most commonly cited psychological outcome measure was self-efficacy with two of the included studie reporting this outcome [13-14]. However, the methods used to assess this psychological construct were different for each study (Table 1). Moreover, the term 'self-efficacy' was used in these studies to reflect individuals' experience with their diabetes, rather than self-efficacy as a general psychological construct. Further condition-specific outcomes reported in the included studies were healthy adjustment after stroke [22] and diabetes distress [15]. One of the aims of this review was to draw conclusions on the impact of peer support for physical health populations, however, the variation in population groups of included studies as well as differences in outcome measurement, make it challenging to systematize findings under one umbrella.

We also examined the impact of timing provision of peer support. O'Dell et al. reported that spinal cord injury patients were not always prepared to process information in the acute stages after injury, therefore consideration needs to be made around when the patient is the most receptive to receiving peer support [21]. No findings within included studies suggest a beneficial impact for the delivery of 'early' peer support on patient outcomes. This is likely to be due to the lack of focus on physical health populations in the acute stage.

\section{Limitations}

This scoping review is limited in terms of the population group considered, namely, those with physical health conditions. Alternative populations that have been shown in the literature to utilize peer support include families and carers of individuals with physical health conditions [29-31]; mental health populations [32-36]; children and young people [37-40]; 'at-risk' groups [41]; veterans [42] and those that are homeless [43].

\section{Conclusions}

In conclusion, this scoping review draws attention to the difficulty in assessing the impact of peer support on general physical health populations due to the heterogeneity in participants, study design, intervention implementation, outcome measures and findings within the included studies. In terms of answering the research question, there appears to be greater consistency in the qualitative methods of the evaluation of peer support as compared to objective methods of evaluation. Synthesis of qualitative findings was grouped into general themes across included studies: (1) peer support leading to a sense of belonging or a sense of community, leading to reduced isolation and feeling understood; (2) peer support as a means of providing and/or consolidating information; (3) peer support as helpful for decision-making; (4) peer support leading to empowerment; and (5) peer support helpful for aiding self-management behaviours. The impact of peer support on objective outcome measures is mixed, with some research suggesting that peer support has a greater impact on those with higher levels of negative psychological affect than those with lower levels. Further research could look to explore this suggestion. Finally, this scoping review has highlighted the need for the piloting of peer support in acute physical health settings in order to bridge the gap that this review has identified in peer support research.

\section{Appendices}

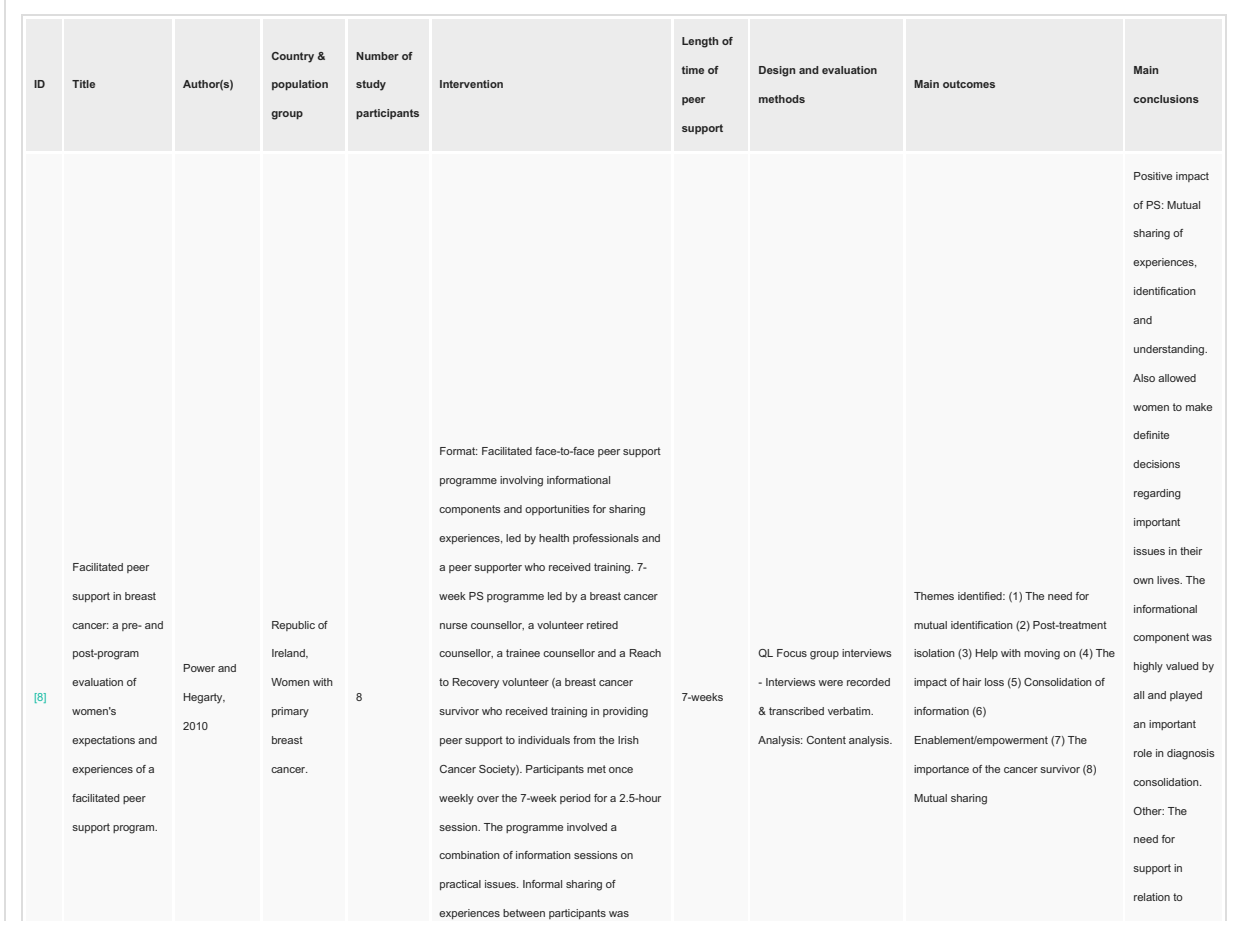




\section{Cureus}

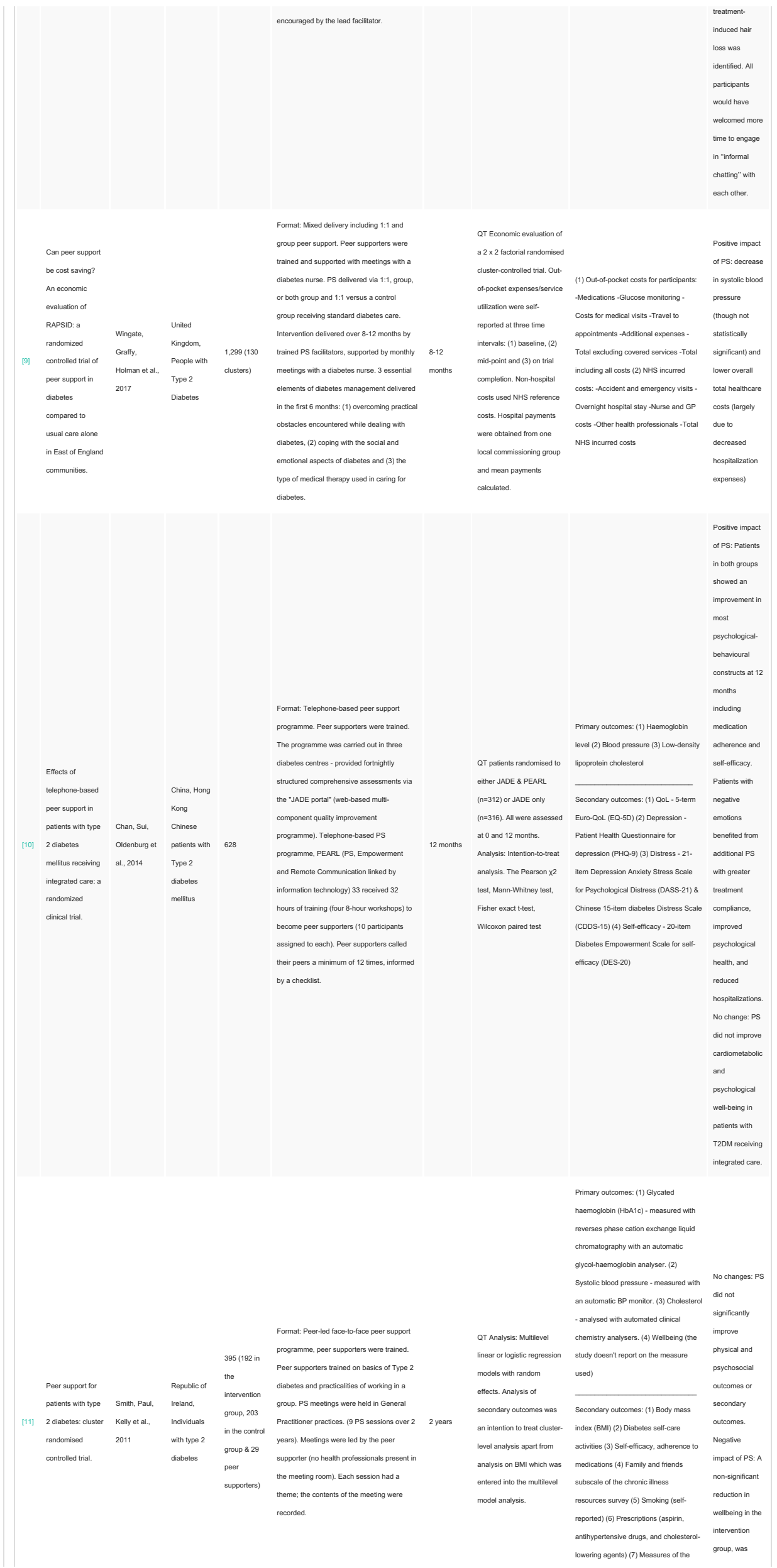




\section{Cureus}

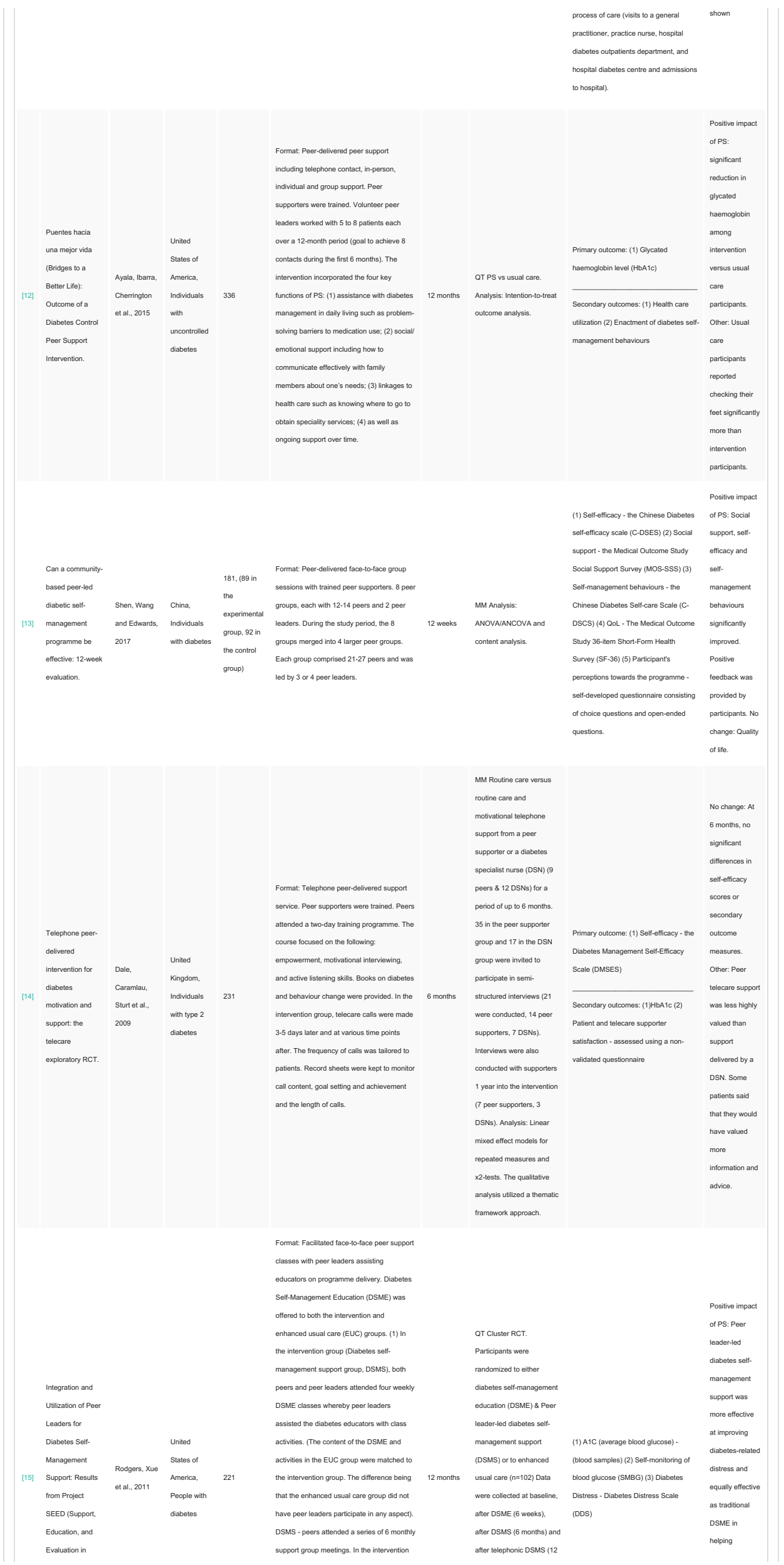




\section{Cureus}

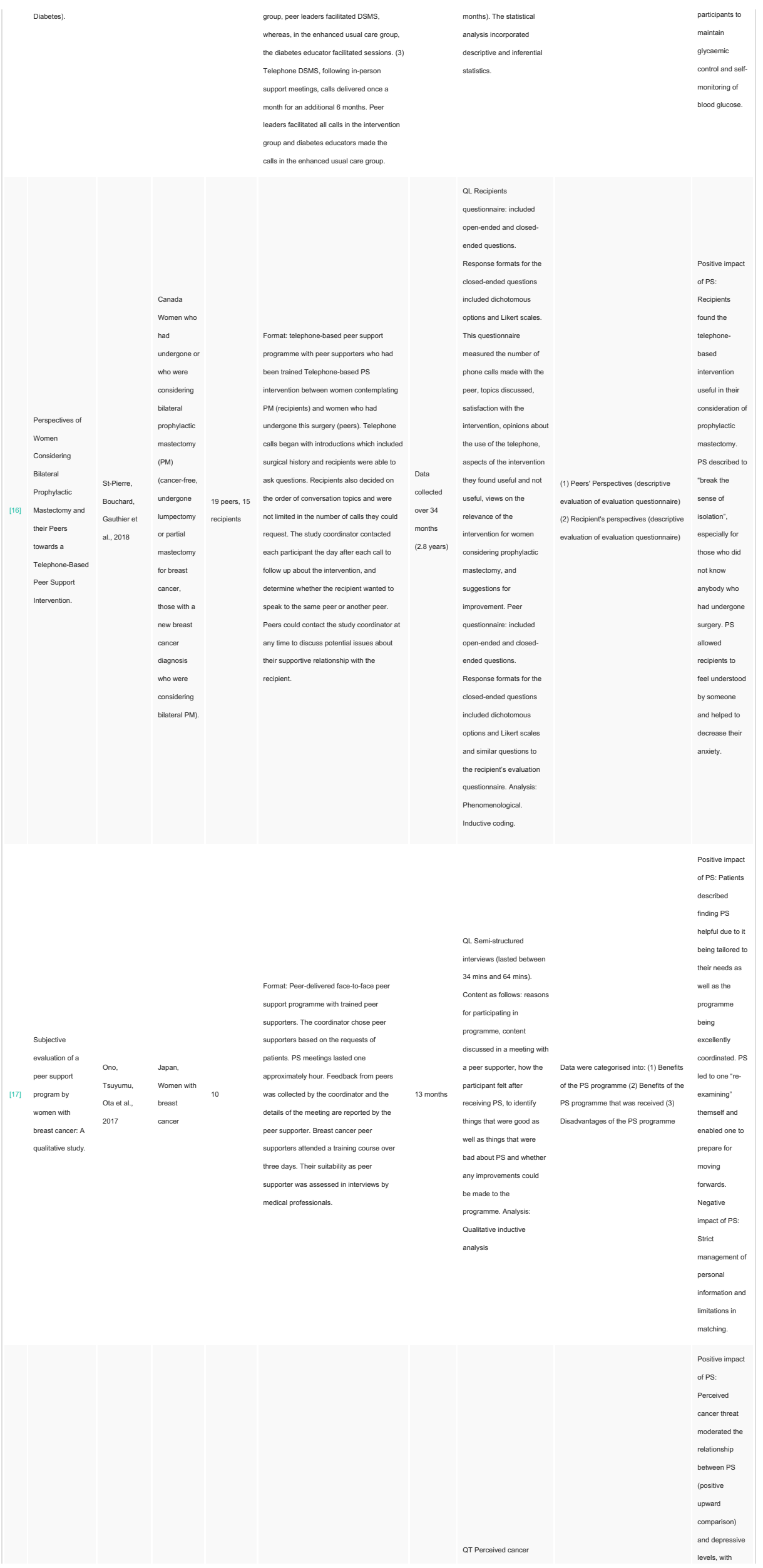




\section{Cureus}

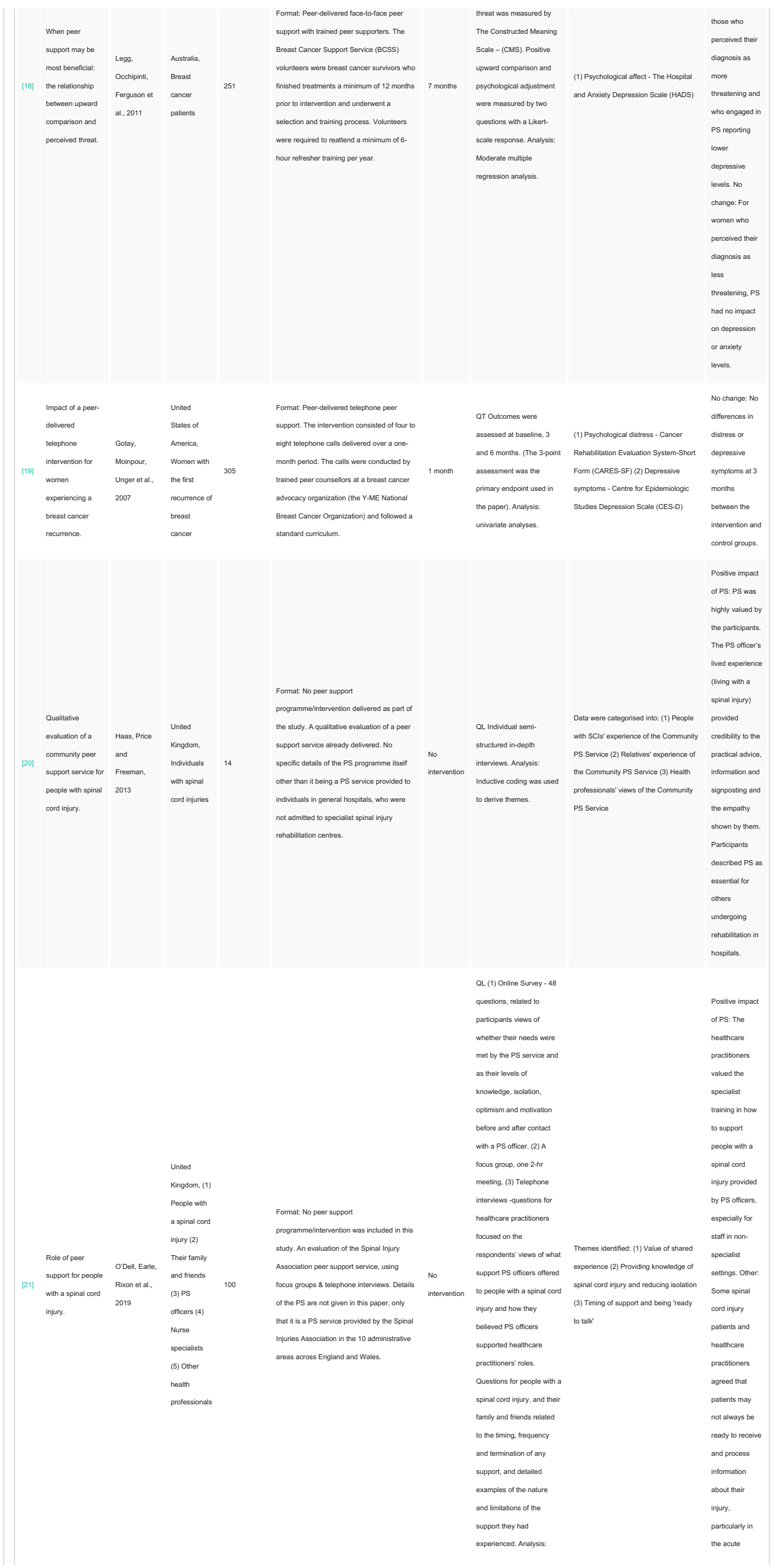




\section{Cureus}

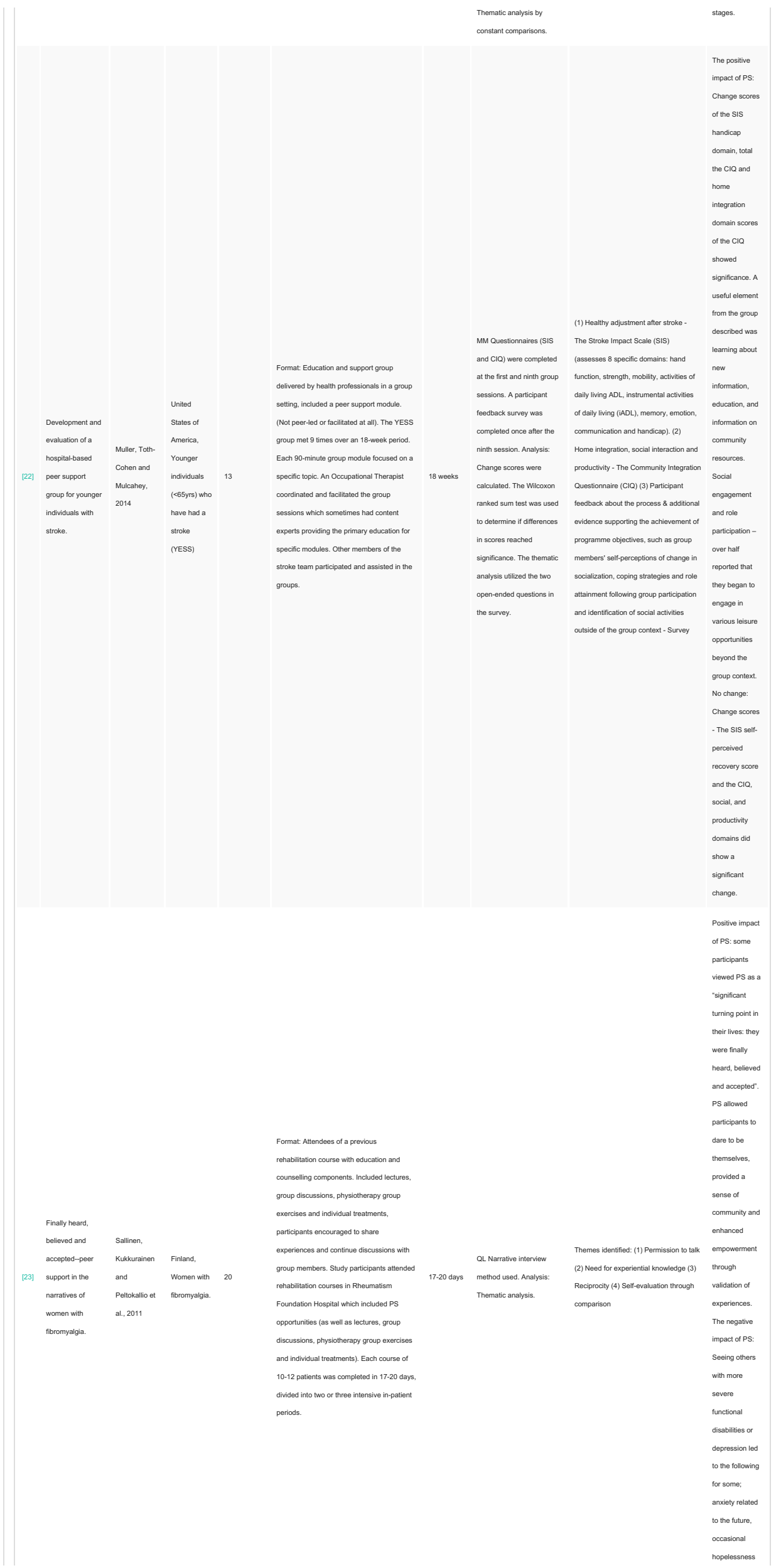




\section{Cureus}

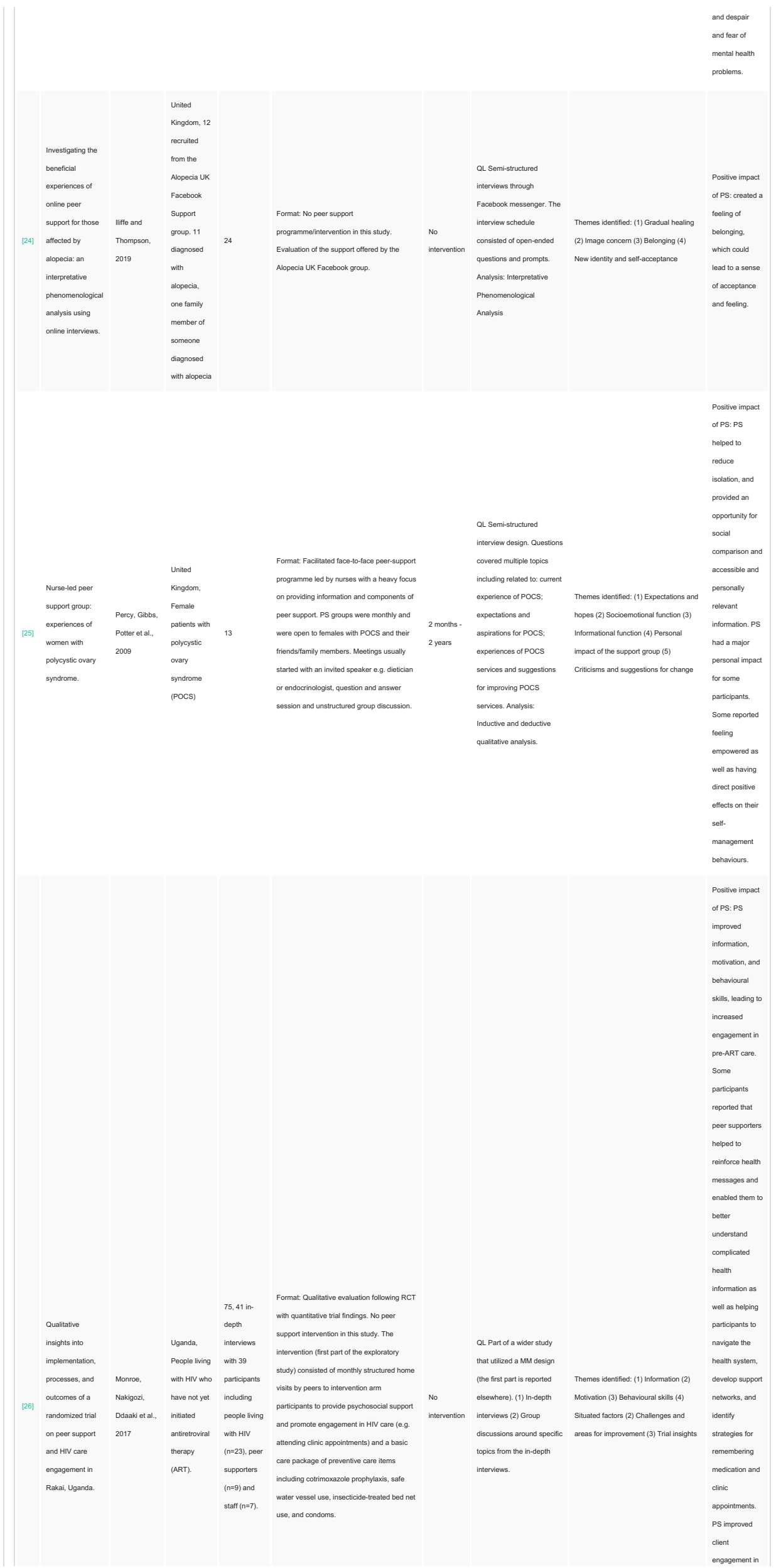




\section{Cureus}

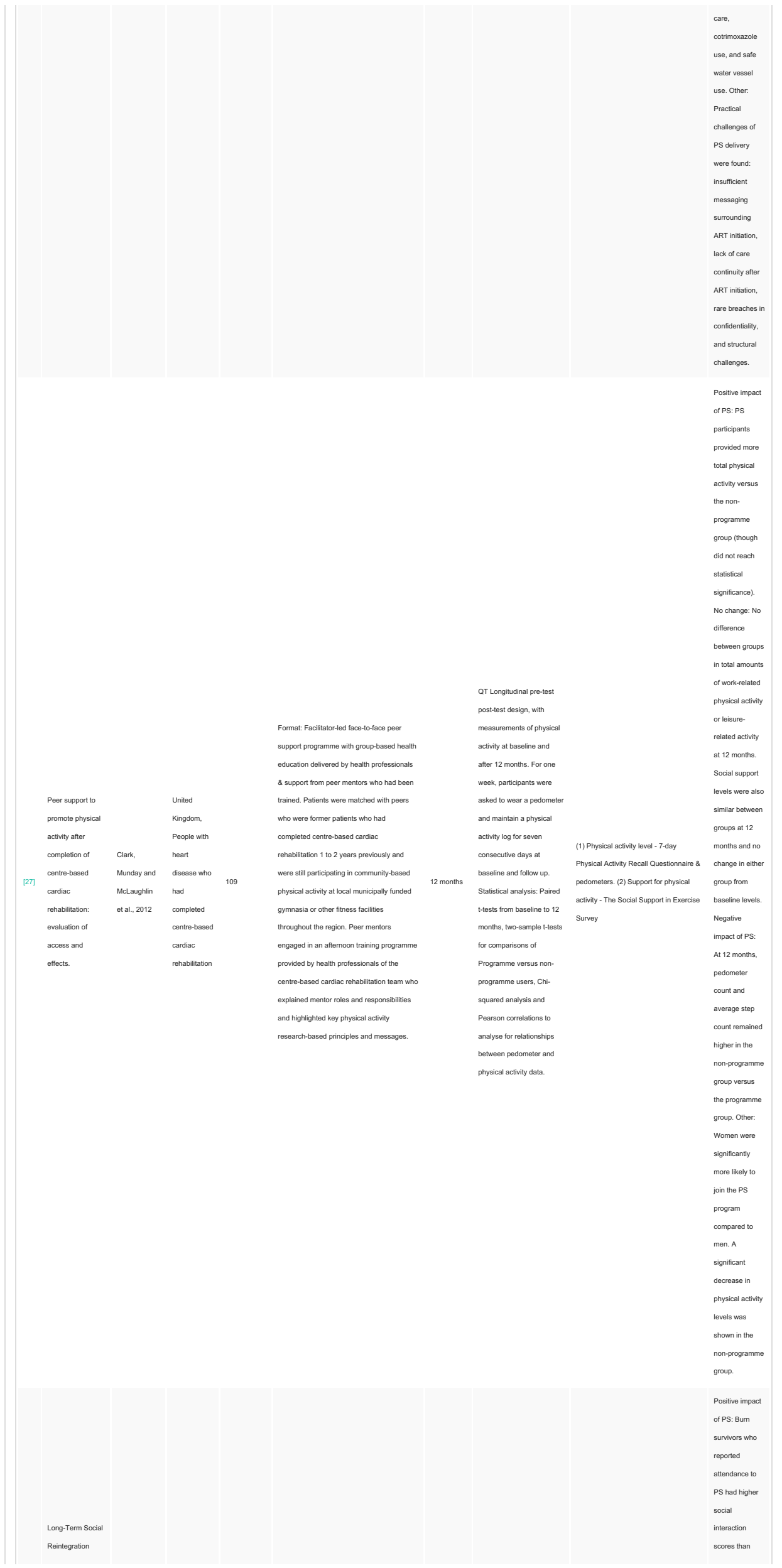




\section{Cureus}

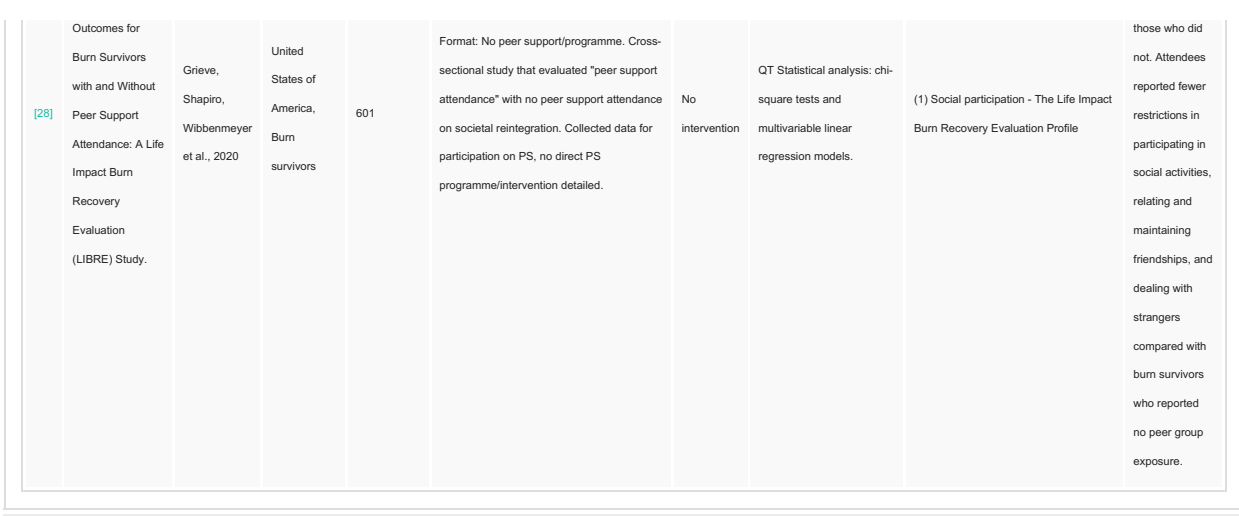

TABLE 3: Characteristics of sources of evidence

RCT: randomized controlled trial; PS: peer support; QT: quantitative methods; QL: qualitative methods; MM: mixed methods

\section{Additional Information}

\section{Disclosures}

Conflicts of interest: In compliance with the ICMJE uniform disclosure form, all authors declare the following: Payment/services info: All authors have declared that no financial support was received from any organization for the submitted work. Financial relationships: All authors have declared that they have no financial relationships at present or within the previous three years with any organizations that might have an interest in the submitted work. Other relationships: All authors have declared that there are no other relationships or activities that could appear to have influenced the submitted work.

\section{References}

1. Graham JT, Rutherford K: The Power of Peer Support. What We Have Learned from the Centre for Socia Innovation Fund. Nesta, London, United Kingdom; 2016.

2. Boothroyd RI, Fisher EB: Peers for progress: promoting peer support for health around the world . Fam Pract. 2010, 27 Suppl 1:i62-8. 10.1093/fampra/cmq017

3. NHS England. The five year forward view for mental health. (2016). https://www.england.nhs.uk/wpcontent/uploads/2016/02/Mental-Health-Taskforce-FYFV-final.pdf.

4. Sokol R, Fisher E: Peer support for the hardly reached: a systematic review . Am J Public Health. 2016, 106:1308. 10.2105/AJPH.2016.303180a

5. National Voices and Nesta. Peer support: what is it and does it work? https://www.nationalvoices.org.uk/sites/default/files/public/publications/peer_support__what_is_it_and_does_it_work.pdf.

6. Wood S, Finnis A, Khan H, et al.: At the Heart of Health. Realising the Value of People and Communities Report. Nesta, London, United Kingdom; 2016.

7. Moher D, Liberati A, Tetzlaff J, Altman DG: Preferred reporting items for systematic reviews and metaanalyses: the PRISMA statement. Ann Intern Med. 2009, 151:264-9, W64. 10.7326/0003-4819-151-4200908180-00135

8. Power S, Hegarty J: Facilitated peer support in breast cancer. A pre- and post-program evaluation of women's expectations and experiences of a facilitated peer support program. Cancer Nurs. 2010, 33:E9-16. 10.1097/NCC.0b013e3181ba9296

9. Wingate L, Graffy J, Holman D, Simmons D: Can peer support be cost saving? An economic evaluation of RAPSID: a randomized controlled trial of peer support in diabetes compared to usual care alone in East of England communities. BMJ Open Diabetes Res Care. 2017, 5:e000328. 10.1136/bmjdrc-2016-000328

10. Chan JC, Sui Y, Oldenburg B, et al.: Effects of telephone-based peer support in patients with type 2 diabetes mellitus receiving integrated care: a randomized clinical trial. JAMA Intern Med. 2014, 174:972-81. 10.1001/jamainternmed.2014.655

11. Smith SM, Paul G, Kelly A, Whitford DL, O'Shea E, O'Dowd T: Peer support for patients with type 2 diabetes: cluster randomised controlled trial. BMI. 2011, 342:d715. 10.1136/bmi.d715

12. Ayala GX, Ibarra L, Cherrington AL, Parada H, Horton L, Ji M, Elder JP: Puentes hacia una mejor vida (Bridges to a Better Life): outcome of a Diabetes Control Peer Support Intervention. Ann Fam Med. 2015, 13 Suppl 1:S9-17. 10.1370/afm.1807

13. Shen H, Wang Y, Edwards H: Can a community-based peer-led diabetic self-management programme be effective: 12-week evaluation. J Clin Nurs. 2017, 26:1621-31. 10.1111/jocn.13526

14. Dale J, Caramlau I, Sturt J, Friede T, Walker R: Telephone peer-delivered intervention for diabetes motivation and support: the telecare exploratory RCT. Patient Educ Couns. 2009, 75:91-8. 10.1016/i.pec. 2008.09.014

15. Piatt GA, Rodgers EA, Xue L, Zgibor JC: Integration and utilization of peer leaders for diabetes selfmanagement support: results from Project SEED (Support, Education, and Evaluation in Diabetes). Diabetes Educ. 2018, 44:373-82. 10.1177/0145721718777855

16. St-Pierre D, Bouchard K, Gauthier L, Chiquette J, Dorval M: Perspectives of women considering bilateral prophylactic mastectomy and their peers towards a telephone-based peer support intervention. I Genet Couns. 2018, 27:274-88. 10.1007/s10897-017-0148-

17. Ono M, Tsuyumu Y, Ota H, Okamoto R: Subjective evaluation of a peer support program by women with breast cancer: a qualitative study. Jpn J Nurs Sci. 2017, 14:38-48. 10.1111/jins.12134

18. Legg M, Occhipinti S, Ferguson M, Dunn I, Chambers SK: When peer support may be most beneficial: the relationship between upward comparison and perceived threat. Psychooncology. 2011, 20:1358-62. 10.1002/pon. 1862

19. Gotay CC, Moinpour CM, Unger JM, et al.: Impact of a peer-delivered telephone intervention for women experiencing a breast cancer recurrence. J Clin Oncol. 2007, 25:2093-9. 10.1200//CO.2006.07.4674

20. Haas BM, Price L, Freeman JA: Qualitative evaluation of a community peer support service for people with spinal cord injury. Spinal Cord. 2013, 51:295-9. 10.1038/sc.2012.14.

21. O'Dell L, Earle S, Rixon A, Davies A: Role of peer support for people with a spinal cord injury . Nurs Stand. 2019, 34:69-75. 10.7748/ns.2018.e10869

22. Muller M, Toth-Cohen S, Mulcahey MJ: Development and evaluation of a hospital-based peer support group for younger individuals with stroke. Occup Ther Health Care. 2014, 28:277-95. 10.3109/07380577.2014.91955

23. Sallinen M, Kukkurainen ML, Peltokallio L: Finally heard, believed and accepted - peer support in the narratives of women with fibromyalgia. Patient Educ Couns. 2011, 85:e126-30. 10.1016/.pec.2011.02.011 


\section{Cureus}

24. Iliffe LL, Thompson AR: Investigating the beneficial experiences of online peer support for those affected by alopecia: an interpretative phenomenological analysis using online interviews. Br J Dermatol. 2019, 181:992-8. 10.1111/bjd.17998

25. Percy CA, Gibbs T, Potter L, Boardman S: Nurse-led peer support group: experiences of women with polycystic ovary syndrome. J Adv Nurs. 2009, 65:2046-55. 10.1111/j.1365-2648.2009.05061. .

26. Monroe A, Nakigozi G, Ddaaki W, et al.: Qualitative insights into implementation, processes, and outcome of a randomized trial on peer support and HIV care engagement in Rakai, Uganda. BMC Infect Dis. 2017, 17:54. 10.1186/s12879-016-2156-0

27. Clark AM, Munday C, McLaughlin D, Catto S, McLaren A, Macintyre PD: Peer support to promote physical activity after completion of centre-based cardiac rehabilitation: evaluation of access and effects. Eur $]$ Cardiovasc Nurs. 2012, 11:388-95. 10.1016/j.ejcnurse.2010.12.001

28. Grieve $B$, Shapiro GD, Wibbenmeyer $L$, et al.: Long-term social reintegration outcomes for burn survivors with and without peer support attendance: a Life Impact Burn Recovery Evaluation (LIBRE) study. Arch Phys Med Rehabil. 2020, 101:S92-8. 10.1016/.apmr.2017.10.007

29. Nicholas DB, Keilty K: An evaluation of dyadic peer support for caregiving parents of children with chronic lung disease requiring technology assistance. Soc Work Health Care. 2007, 44:245-59. 10.1300//010v44n03_08

30. Nicholas DB, Chahauver A, Brownstone D, Hetherington R, McNeill T, Bouffet E: Evaluation of an online peer support network for fathers of a child with a brain tumor. Soc Work Health Care. 2012, 51:232-45. 10.1080/00981389.2011.631696

31. Lammers EJ, Zickafoose IS, Peterson GG, Blue L, Stewart KA, Kranker K: Parent partners: evaluation of a novel peer-support intervention for the caregivers of children hospitalized for behavioral health conditions. Acad Pediatr. 2019, 19:908-16. 10.1016/j.acap.2019.06.001

32. Castelein S, Bruggeman R, van Busschbach IT, van der Gaag M, Stant AD, Knegtering H, Wiersma D: The effectiveness of peer support groups in psychosis: a randomized controlled trial. Acta Psychiatr Scand. 2008 , 118:64-72. 10.1111/j.1600-0447.2008.01216.X

33. Hanly F, Torrens-Witherow B, Warren N, et al.: Peer mentoring for individuals with an eating disorder: qualitative evaluation of a pilot program. J Eat Disord. 2020, 8:29. 10.1186/s40337-020-00301-8

34. Scanlan IN, Hancock N, Honey A: Evaluation of a peer-delivered, transitional and post-discharge support program following psychiatric hospitalisation. BMC Psychiatry. 2017, 17:307. 10.1186/s12888-017-1469-x

35. Aschbrenner KA, Naslund JA, Shevenell M, Kinney E, Bartels SJ: A pilot study of a peer-group lifestyle intervention enhanced with mHealth technology and social media for adults with serious mental illness. J Nerv Ment Dis. 2016, 204:483-6. 10.1097/NMD.0000000000000530

36. Sheehan L, Torres A, Lara JL, et al.: Qualitative evaluation of a peer navigator program for Latinos with serious mental illness. Adm Policy Ment Health. 2018, 45:495-504. 10.1007/s10488-017-0839-5

37. Webster RA, Hunter M, Keats JA: Evaluating the effects of a peer support programme on adolescents knowledge, attitudes and use of alcohol and tobacco. Drug Alcohol Rev. 2002, 21:7-16. $10.1080 / 09595230220119282$

38. Kew KM, Carr R, Crossingham I: Lay-led and peer support interventions for adolescents with asthma Cochrane Database Syst. Rev. 2017, 4:CD012331. 10.1002/14651858.cd012331

39. Patterson P, McDonald F, Tindle R, Kelly-Dalgety E, Zebrack B, Costa D: The development and preliminary evaluation of the Cancer Peer Support Scale in adolescents living with cancer. Psychooncology. 2018, 27:2865-8. 10.1002/pon.4869

40. Thupayagale-Tshweneagae G, Mokomane Z: Evaluation of a peer-based mental health support program for adolescents orphaned by AIDS in South Africa. Jpn J Nurs Sci. 2014, 11:44-53. 10.1111/1.17427924.2012.00231.x

41. Janssen PA, Gibson K, Bowen R, Spittal PM, Petersen KL: Peer support using a mobile access van promotes safety and harm reduction strategies among sex trade workers in Vancouver's Downtown Eastside. J Urban Health. 2009, 86:804-9. 10.1007/s11524-009-9376-1

42. Jain S, Hernandez JM, Lindley SE: Peer support program for veterans in rural areas . Psychiatr Serv. 2014, 65:1177. 10.1176/appi.ps.650704

43. Miler JA, Carver H, Foster R, Parkes T: Provision of peer support at the intersection of homelessness and problem substance use services: a systematic 'state of the art' review. BMC Public Health. 2020, 20:641. $10.1186 /$ s12889-020-8407-4 